\title{
Pollen Allergens for Molecular Diagnosis
}

\author{
Isabel Pablos ${ }^{1} \cdot$ Sabrina Wildner $^{2}$ - Claudia Asam ${ }^{1}$ • Michael Wallner ${ }^{1} \cdot$ \\ Gabriele Gadermaier ${ }^{1,2}$
}

Published online: 22 March 2016

(C) The Author(s) 2016. This article is published with open access at Springerlink.com

\begin{abstract}
Pollen allergens are one of the main causes of type I allergies affecting up to $30 \%$ of the population in industrialized countries. Climatic changes affect the duration and intensity of pollen seasons and may together with pollution contribute to increased incidences of respiratory allergy and asthma. Allergenic grasses, trees, and weeds often present similar habitats and flowering periods compromising clinical anamnesis. Molecule-based approaches enable distinction between genuine sensitization and clinically mostly irrelevant $\operatorname{IgE}$ cross-reactivity due to, e. g., panallergens or carbohydrate determinants. In addition, sensitivity as well as specificity can be improved and lead to identification of the primary sensitizing source which is particularly beneficial regarding polysensitized patients. This review gives an overview on
\end{abstract}

This article is part of the Topical Collection on Immunologic/Diagnostic Tests in Allergy

Gabriele Gadermaier

Gabriele.Gadermaier@sbg.ac.at

Isabel Pablos

Isabel.Pablos@sbg.ac.at

Sabrina Wildner

Sabrina.Wildner@sbg.ac.at

Claudia Asam

Claudia.Asam@sbg.ac.at

Michael Wallner

Michael.Wallner@sbg.ac.at

1 Department of Molecular Biology, University of Salzburg, Hellbrunner Straße 34, 5020 Salzburg, Austria

2 Christian Doppler Laboratory for Biosimilar Characterization, University of Salzburg, Hellbrunner Straße 34, 5020 Salzburg, Austria relevant pollen allergens and their usefulness in daily practice. Appropriate allergy diagnosis is directly influencing decisions for therapeutic interventions, and thus, reliable biomarkers are pivotal when considering allergen immunotherapy in the context of precision medicine.

Keywords Pollen allergens · Molecule-based diagnosis · Grass pollen allergens $\cdot$ Tree pollen allergens $\cdot$ Weed pollen allergens

\section{Introduction}

Allergic reactions to pollen represent the most frequent type I allergies affecting up to $30 \%$ of the industrialized population (www.eaaci.org). Climatic changes are expected to influence the duration as well as the intensity of pollen seasons which might in hand with air pollution contribute to increased numbers of respiratory allergy and asthma $[1,2]$. In addition, pollen-derived nanovesicles and other small components (e.g., adenosine) may play a specific role in the course of allergic diseases [3, 4]. Clinical anamnesis of pollen allergies to identify the disease-eliciting source can be hampered by similar habitat and flowering periods of certain plants and the fact that symptoms may be elicited by pollen transported far by the wind [5]. In addition, patients are often multi-sensitized to diverse allergen sources due to IgE cross-reactivity, co-sensitization, or both [6-10]. Skin prick tests and specific $\operatorname{IgE}$ detection using crude pollen extracts are currently performed in routine allergy diagnosis. Allergen extracts however contain a variety of allergenic and non-allergenic components, and standardization of pollen extracts is difficult due to varying source material and product preparations [11]. Besides source specific and thus genuine marker allergens, also minor allergens including panallergens as wells as allergens with cross- 
reactive carbohydrates (CCDs) are present in diagnostic extracts which might impede exact diagnosis. Molecular diagnosis using well-characterized purified allergen components from natural source or produced as recombinant molecule allows clinicians to obtain detailed information on sensitization profiles and thus supports improved patients' management $[11,12 \bullet]$.

More than 150 pollen allergens are officially acknowledged by the IUIS allergen nomenclature sub-committee originating from grasses, trees, and weeds (www.allergen.org). This review focuses on the most relevant and commercially available components which are discussed in more detail (Tables 1 and 2). We also provide an overview on diverse IgE cross-reactivity profiles within the Ole e 1-like, pectate lyase and non-specific lipid transfer protein (nsLTP) families (Fig. 1).

\section{Methods for Molecule-Based Allergy Diagnosis}

Apart from clinical history, skin prick tests (SPT) using allergen extracts represent one of the most common used in vivo diagnostic tools to confirm an immediate IgE-mediated allergic reaction. Since the 1990s, the performance of several purified recombinant pollen allergens was investigated in provocation tests and compared to natural allergens and extracts [13•]. Though refinement of diagnosis with purified molecules was proven effective, in vivo diagnosis using components is nowadays widely restricted to GMP-produced material. Alternatives such as the basophil activation test (BAT), an in vitro method to monitor upregulation of activation markers CD63 and CD203 upon allergen-triggered activation of primary basophils, or mediator release assays with passively sensitized basophils, which measure either histamine or $\beta$-hexosaminidase, may help to circumvent this problem. While these assays demonstrate functional allergen responses, they are rather demanding regarding the costs and experimental skills and thus are not used in daily practice. Measurement of specific $\operatorname{IgE}(\operatorname{sgE})$ in serum is currently the most widely used in vitro method for allergy diagnosis. Besides allergen extracts provided for singleplex analyses (ImmunoCAP), a panel of purified natural and recombinant components is commercially available for routine diagnosis. In addition, allergen microarrays with more than 100 purified allergen molecules (ImmunoCAP ISAC) enable simultaneous IgE measurement using only minute amounts of blood. These multiplex assays offer an attractive alternative to refine allergy diagnosis, monitor disease progression as well as therapeutic outcomes [14]. All in vitro methods provide reliable information on $\operatorname{IgE}$ sensitization while correlation with clinically relevant symptoms remains challenging [15].

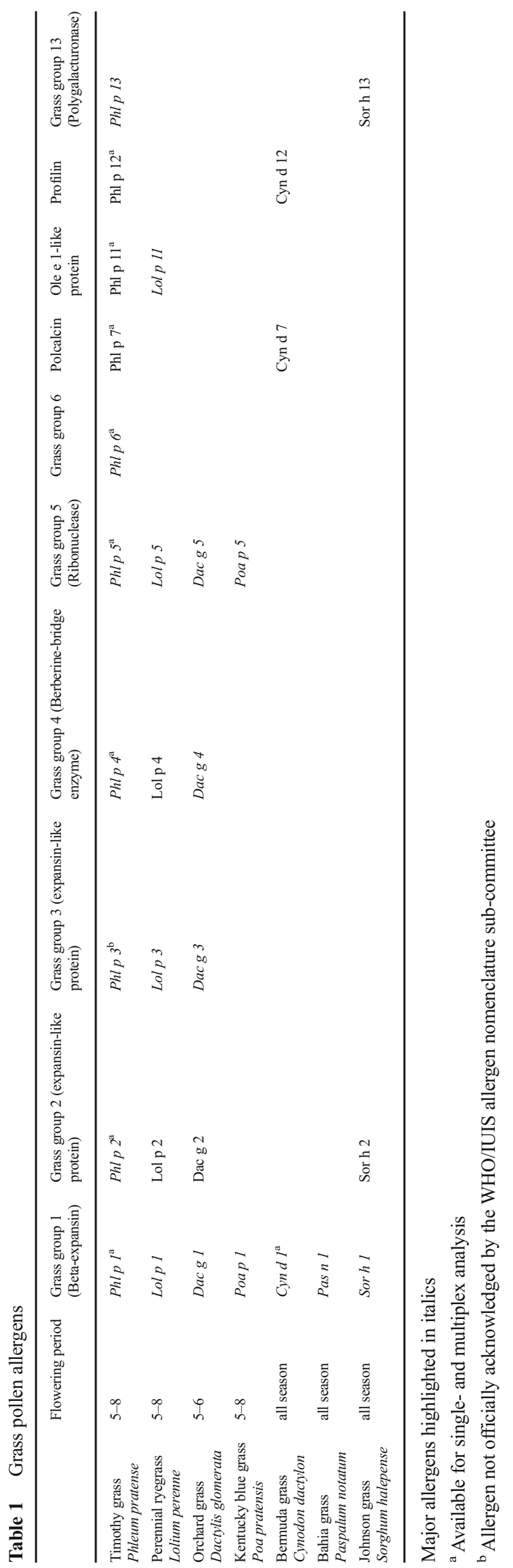




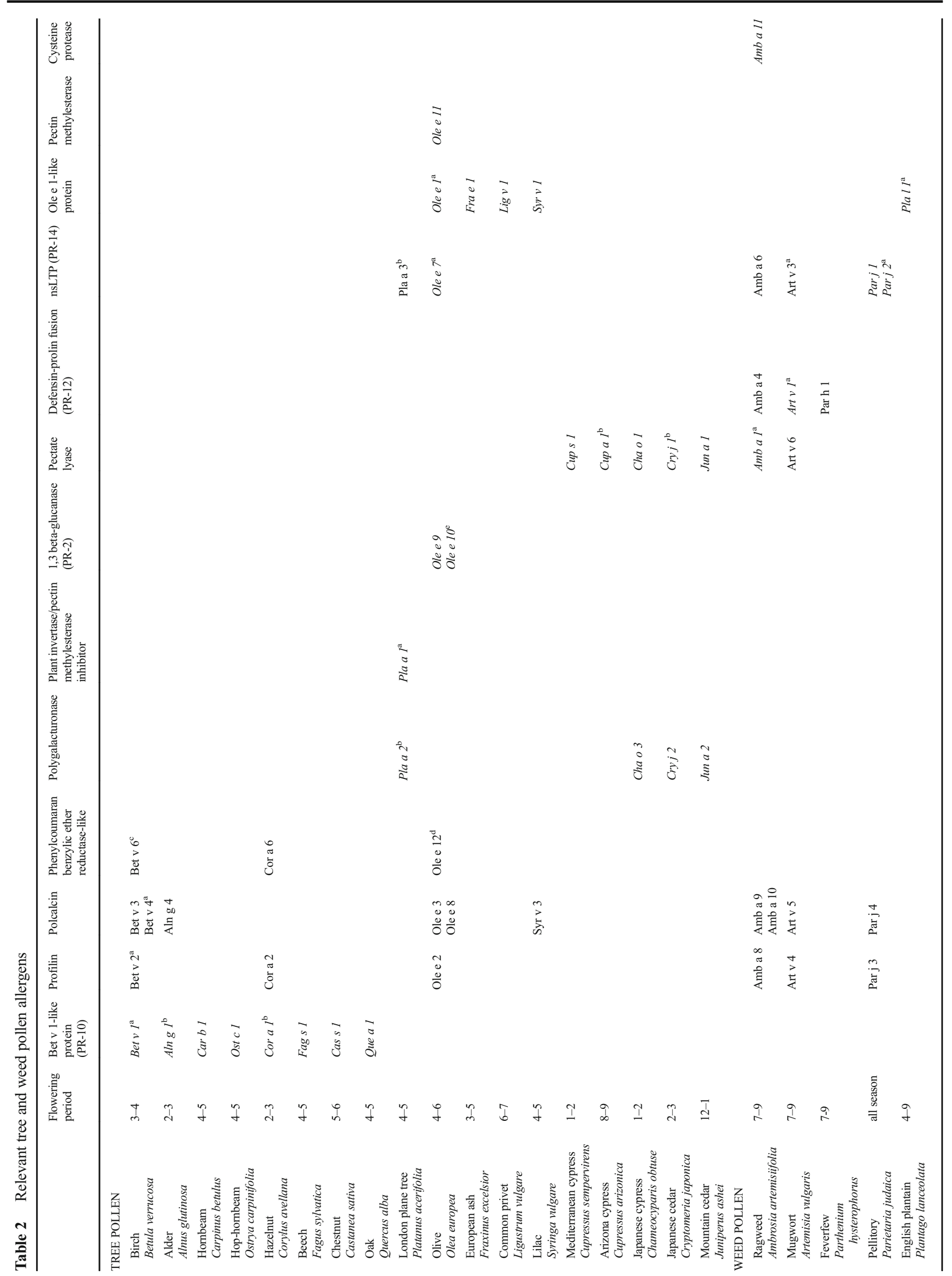




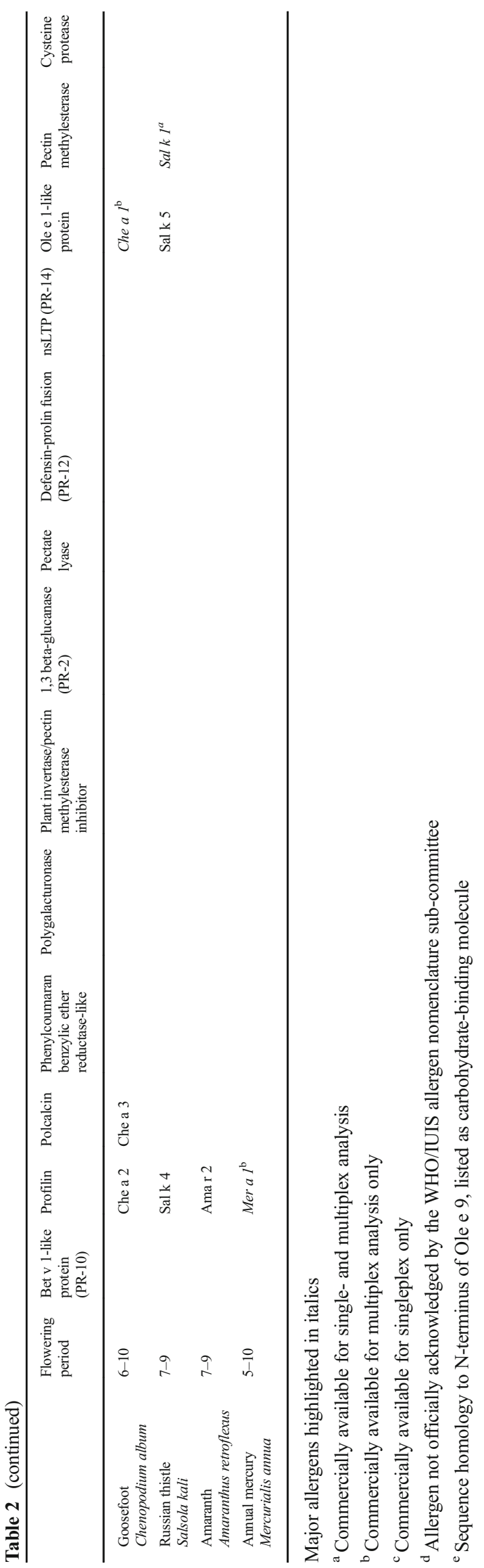

a Ole e 1-like family

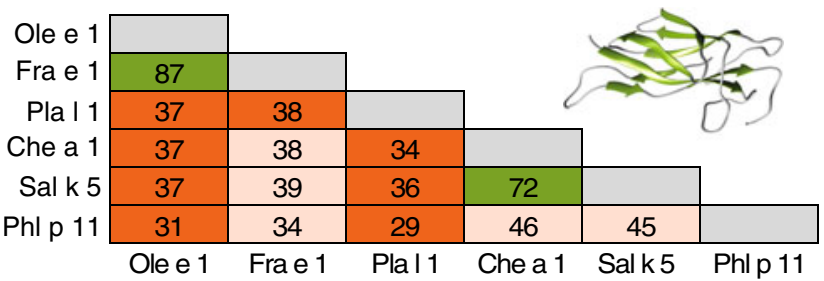

b Pectate lyases

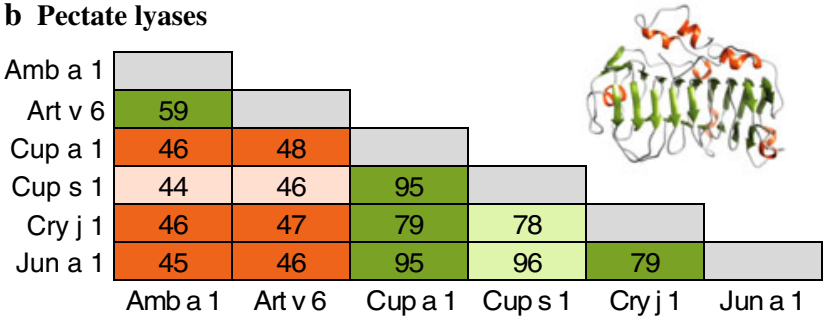

c Lipid transfer proteins

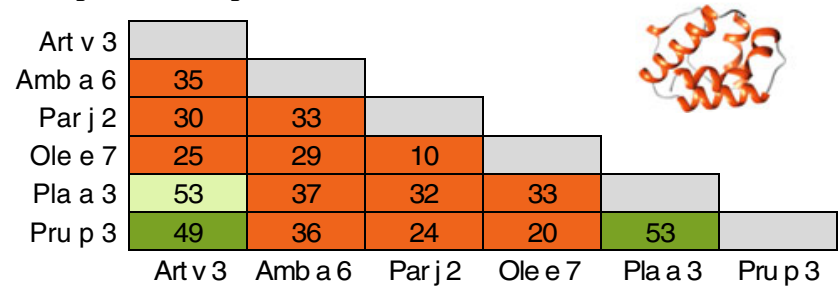

Fig. 1 Sequence identity matrix and 3D-models of allergenic protein families. a Ole e 1-like proteins and structure of Pla 11.0101 (4Z8W), b pectate lyases and model of Amb a 1.0101 (template 1PXZ), and $\mathbf{c}$ lipid transfer proteins and model of Art v 3.0201 (template 2B5S). Multiple sequence alignments performed in Clustal Omega. Models were generated using Swiss-Model (www.swissmodel.expasy.org), and ribbon cartons are shown using UCSF Chimera (www.cgl.ucsf.edu/chimera). Green boxes represent demonstrated $\mathrm{IgE}$ cross-reactivity, light green boxes represent potential IgE cross-reactivity based on high sequence identity, red boxes represent no/limited demonstrated IgE cross-reactivity and light red boxes represent no/limited IgE cross-reactivity based on low sequence identity

\section{Grass Pollen Allergens}

Grasses are cultivated for food, animal fodder, surface vegetation, and meadows, but also represent one of the major causes of respiratory allergies worldwide [16-19]. The clinically most relevant source of pollen allergens is found within the Poaceae family. Among this, the Pooideae subfamily is prevalent in temperate climates and the most abundant allergenic species within the Pooideae are Timothy grass, Perennial ryegrass, Orchard grass, and Kentucky blue grass. The pollination season typically starts in spring and lasts until late summer, pollen peaks are usually found between June and July. In subtropical regions, members of the Chloridoideae and Panicoideae subfamilies are widely distributed. Here, the most important species triggering pollen allergy are Bermuda grass, Bahia grass, and Johnson grass [20]. 
Pollination of these grasses persists throughout the year peaking from early summer through autumn. Climatological changes due to global warming and human activities have additionally influenced pollen distribution as well as potency which further increases sensitization risks $[21 \bullet \bullet]$.

\section{Timothy Grass}

Timothy grass is widely distributed in the temperate climate and one of the best characterized allergenic grasses. The highest sensitization prevalence is registered in the European population, ranging from 18.5 to $28.5 \%$ [6]. A prevalence of $36 \%$ among Austrian pupils was recently found using allergen microarray analysis (Stemeseder et al., unpublished). The majority of commercially available components for grass pollen allergy originate from Timothy grass (Table 1). In this context, Phl p 1, a major allergen from Timothy grass with a sensitization prevalence of $>90 \%$ among grass pollen allergic patients, seems extremely important [22, 23]. Phl p 1-specific $\operatorname{IgE}$ is considered a marker for genuine sensitization and was recently also proposed as "initiator molecule" for Timothy grass pollen allergy $[21 \bullet \bullet, 24 \cdot \bullet]$. In the study by Hatzler et al., $75 \%$ of grass pollen allergic patients started with mono-sensitization to $\mathrm{Phl} \mathrm{p} \mathrm{1,} \mathrm{in} \mathrm{some} \mathrm{cases} \mathrm{years} \mathrm{before}$ onset of clinical symptoms, which subsequently developed into poly-sensitization towards other grass pollen allergens $[24 \bullet \bullet$.

The second major allergen in this source is Phl p 5, triggering specific IgE in $65-90 \%$ of grass pollen allergic patients. Together with Phl p 1, it is considered a marker molecule for grass pollen allergy, and in rare cases of absent $\mathrm{Phl} p 1$-specific $\mathrm{IgE}, \mathrm{Phl} \mathrm{p} 5$ is giving reliable results of genuine sensitization $[21 \bullet \bullet, 22]$. Phl p 5 is highly cross-reactive with other group 5 grass pollen allergens, which are restricted to grasses of the Pooideae subfamily $[25 \cdot, 26]$. A high sequence homology with Phl p 6 is observed, and significant levels of $\operatorname{IgE}$ crossreactivity have been demonstrated [26]. Phl p 6 is frequently used together with $\mathrm{Phl} \mathrm{p} 2$ and Phl p 5 to confirm specific sensitization to Pooideae grasses $[21 \bullet \bullet]$. However, care should be taken with interpretation of $\mathrm{Phl} p 2$ results since a homologous molecule termed Sor h 2 was recently identified in Johnson grass, a member of the Panicoideae subfamily [25.]. The tentatively termed Timothy grass pollen allergen $\mathrm{Phl} \mathrm{p} 3$ shows sequence identity with $\mathrm{Phl} \mathrm{p} 2$ and partially with the $\mathrm{C}$-terminus of Phl $\mathrm{p} 1$; however, while crossreactivity between group 2 and 3 grass pollen allergens was demonstrated, relevant cross-reactivity to group 1 allergens is not evident [27].

Among grass pollen components, solely $\mathrm{Phl} p 4$ is provided as natural protein harboring CCDs. Recombinant Phl p 4 demonstrates lower IgE reactivity, thus IgE-binding to commercially available natural $\mathrm{Phl} \mathrm{p} 4$ should be analyzed for CCDs reactivity [7, 28]. In Timothy grass, the panallergens Phl p 7 (polcalcin) and $\mathrm{Phl} \mathrm{p} 12$ (profilin) are present probably inducing IgE cross-reactivity with homologs from trees, weeds and foods [29]. In the absence of Phl p 1- and/or $\mathrm{Phl} \mathrm{p}$ 5-specific IgE, sensitization to panallergens is not indicative of genuine grass pollen reactivity [21••]. Nevertheless, an association of the Phl p 12 sensitization and the development of oral allergy syndromes were demonstrated in Italian children [30]. Due to the low sequence similarity, IgE cross-reactivity of $\mathrm{Phl} p 11$ with other Ole e 1-like family members from trees and weeds is absent or very limited [29] (Stemeseder et al., unpublished).

\section{Perennial Ryegrass, Orchard Grass, and Kentucky Blue Grass}

Allergens from Perennial ryegrass, Orchard grass, and Kentucky blue grass have been identified and are listed in Table 1, but none of those is commercially available for diagnosis. Due to high sequence identity and extensive IgE crossreactivity of allergens within the Pooideae subfamily, diagnosis to temperate grasses is usually performed with components from Timothy grass $[21 \bullet \bullet, 25 \bullet, 26,31]$.

\section{Bermuda Grass}

Cyn $\mathrm{d} 1$, the major allergen from Bermuda grass, is currently the only commercially available molecule of the subtropical grasses. It is considered as biomarker for genuine sensitization to the Chloridoideae subfamily [21••]. Cyn $\mathrm{d} 1$ is recognized by $76-100 \%$ of grass pollen allergic patients, which may also result in IgE cross-reactivity with $\mathrm{Phl} p 1$ [31]. Therefore, it was suggested that the primary sensitization to Bermuda grass is indicated when specific IgE-levels to Cyn d 1 exceed IgEbinding to $\mathrm{Phl} \mathrm{p} 1$ and IgE to $\mathrm{Phl} p 5$ is not detectable. Since Cyn $\mathrm{d} 1$ is harboring CCDs, glycan-derived reactivity should be monitored during diagnosis [7].

\section{Bahia Grass and Johnson Grass}

Currently, no allergen from the Panicoideae subfamily is commercially available for molecular diagnosis. In recent years, a growing number of allergens from these sources have been described. Timbrell et al. showed improved sensitivity and specificity when using $\mathrm{nPas} \mathrm{n} 1$ for molecular diagnosis of Bahia grass pollen allergic patients [32•]. The authors suggested to include the Pas $\mathrm{n} 1$ for commercially available diagnosis of Bahia grass allergy as a sub-group of patients showed species-specific IgE and T cell reactivity [33, 34]. As mentioned above, Sor h 2 was recently identified in Johnson grass which refutes the assumption that group 2 allergens are absent in subtropical grasses $[21 \bullet \bullet, 25 \bullet, 35]$. 


\section{Tree Pollen Allergens}

In general, trees are woody perennial plants with an elongated self-supporting stem or trunk and supporting branches that form a more or less defined crown. To distinguish trees from shrubs, a certain stem height or diameter is sometimes used as decision criteria. Trees do not comprise a single taxonomic group and include various species that separately evolved stems and branches [36]. Most of the clinically relevant pollen allergens are produced by wind-pollinated trees belonging to only four different orders, which show an almost worldwide distribution. For details on tree pollen allergens, review Asam et al. [37••].

\section{Birch and Related Fagales Species}

Classified within the order of Fagales, birch and the related tree species alder, hornbeam, hop-hornbeam, hazelnut, beech, chestnut, and oak constitute the main cause of early seasonal rhinitis in the temperate climate zone of the Northern Hemisphere [8]. Worldwide, more than 100 million patients suffer from birch pollen allergies and in Europe clinically relevant sensitization to birch affects around $19.6 \%$ of the allergic population. Clinically relevant sensitization rates to hazel and alder pollen were reported at 17.1 and $16.2 \%$, respectively $[38 \bullet \bullet, 39]$. The major allergens from Fagales trees are classified as members of the pathogenesis-related-10 (PR10) proteins, and Bet $v 1$ from birch pollen is generally acknowledged as main sensitizer and marker allergen of this family (Table 2). Among birch pollen allergic individuals, sensitization rates to Bet $\mathrm{v} 1$ of up to $90 \%$ or more have been reported [40, 41]. However, inhibition experiments revealed that besides birch, several other Fagales species might have the potential to sensitize susceptible individuals [9]. This seems especially important in areas where birch trees are virtually absent, thus exposure to other allergenic Fagales pollen will eventually lead to sensitization and the development of Fagales pollen allergy. Currently, Bet v 1 is the only group 1 Fagales allergen offered for singleplex, while Aln g 1 and Cor a 1 are additionally available for multiplex analysis. Since oak populations are very wide-spread not only in Europe but also in vast parts of North America and Asia and do not necessarily coexist with birch or hazel, Que a 1 should be considered for diagnosis especially in those areas. More than $70 \%$ of birch pollen allergic patients develop adverse symptoms to food such as fruits, nuts, or vegetables [42]. This clinical condition often referred to as pollen-food syndrome always involves a pre-sensitization to Fagales pollen allergens [43]. Therefore, diagnosis of Bet $v$ 1-related food allergies is directly linked to a correct diagnosis of the underlying pollen allergy. Moreover, several minor allergens, among them the panallergens profilin and polcalcin, have been identified in many allergenic
Fagales species, evoking sensitization rates of 44.6 and $9.4 \%$, respectively [44].

\section{Plane Tree}

Plane trees belong to the order of Proteales and are native to the Northern Hemisphere. They preferentially grow in temperate regions from Asia to Europe and North America. Due to their resistance towards pollution, they are widely used as ornamental trees in urban areas and at roadsides $[45,46]$. With $3.3 \%$ in the allergic population, clinically relevant sensitization throughout Europe is rather low but can reach almost $12 \%$ in the UK [38*0]. More than $80 \%$ of plane-sensitized patients react to the major allergens Pla a 1 (plant invertase inhibitor) and Pla a 2 (polygalacturonase) [45, 47, 48]. The minor allergen Pla a 3 is a member of the nsLTP family and shows high cross-reactivity with Pru p 3, the major allergen from peach [49].

\section{Olive, Ash, Lilac, and Privet}

The major allergy-eliciting species within the order of Lamiales are olive, ash, lilac, and privet. Olive trees are preferentially cultivated in the Mediterranean area where up to $70 \%$ of patients with respiratory symptoms are sensitized to olive pollen [8]. With a sensitization prevalence of $80 \%$ among olive pollen allergic patients, Ole e 1 represents the major allergen and thus is the eponym of the Ole e 1-like protein family with homologs in tree, grass, and weed pollen. While cross-reactivity to homologues in other Oleaceae trees is very high, cross-reactivity with grass $(\mathrm{Phl} \mathrm{p} \mathrm{11)} \mathrm{and} \mathrm{weed}$ (e.g., Pla 1 1) Ole e 1-like allergens is limited [37••] (Stemeseder et al., unpublished). Besides Ole e 1, 10 other olive pollen allergens, including the panallergens Ole e 2 (profilin) and Ole e 3 (polcalcin), have been described and details can be reviewed in Villalba et al. [50•]. Depending on geographic location and thus on the exposure level of the population, the allergens Ole e $6,7,10$, and 11 may eventually become major allergens of olive pollen [50•, 51].

In central Europe, ash pollen may cause sensitization rates from 4 to $18 \%$ [37••]. The major allergen of European ash, Fra e 1, has been classified as member of the Ole e 1-like family and shows high sequence similarity to other Ole e 1like proteins from Lamiales [52]. A recent study from Imhof et al. confirmed the high cross-reactivity between Ole e 1 and Fra e 1 and suggested using Ole e 1 as diagnostic maker of ash pollen allergy [53]. Moreover, a $\beta$-1,3-glucanase from ash termed Fra e 9 was recently identified, which can account for a sensitization prevalence up to $60 \%$ in distinct areas of France [54]. Common privet as well as lilac are often used for ornamental purposes and frequently found in Europe, Asia, and North America. Both express highly cross-reactive major allergens belonging to the Ole e 1-like protein family (Lig v 1 
and Syr v 1, respectively); sensitization rates are however generally low [55].

\section{Allergenic Cypress Species, Japanese Cedar, and Juniper Trees}

The order of Pinales comprises allergenic cypress, cedar as well as juniper species. In contrast to the previously described allergenic trees, Pinales are gymnosperms widely distributed throughout the Northern Hemisphere. Mediterranean cypress and its American relative, Arizona cypress, which is primarily native to the south-west of North America, are closely related to evergreen trees which coexist in the Mediterranean area (www.eol.org). Among the allergic individuals in Europe, 2. $6 \%$ show a clinically relevant sensitization to cypress, which is generally low, however, may reach levels of up to $42.7 \%$ as reported for allergic patients in Italy [56]. The major allergens of both species, Cup s 1 and Cup a 1, belong to the pectate lyase family and share $95.1 \%$ sequence identity. Moreover, clinically relevant, highly cross-reactive, allergenic pectate lyases have been identified as major allergens in Mountain cedar (Jun a 1) endemic in the USA, as well as in Japanese cypress (Cho o 1) and Japanese cedar (Cry j 1). The latter two species form dominant populations on the Japanese island [37••]. Sensitization rates evoked by Cha o 1 or the more potent Cry j 1 affect up to $40 \%$ of the population in certain areas of Japan [57, 58], whereas sensitization to Jun a 1 represents a serious health risk in Texas and Mexico [59]. Moreover, the polygalacturonases Jun a 2, Cry j 2 and Cha o 2 , have been identified as major allergens in these Cupressaceae species being responsible for sensitization rates of up to $80 \%[37 \bullet \bullet, 60,61]$. Nevertheless, allergenic polygalacturonases did not get much attention so far but should eventually be considered as candidates for allergy diagnosis.

\section{Weeds Pollen Allergens}

The term "weed" does not refer to any specific botanical family but rather describes plants outside the order of trees or grasses. They are used as culinary herbs, medicinal plants, and are frequently ecologically adaptive segetal plants [62]. Climatic changes are generally impacting the flora, which might be particularly advantageous for weeds as they are able to dominate groundcover, adapt to various environments, or reside in ecologic niches. Weed pollen allergic patients are frequently polysensitized to diverse plant sources, thus molecule-based approaches are especially valuable for precise diagnosis. A comprehensive overview on production and botanical classification of weed pollen allergens is provided in Gadermaier et al. and Villalba et al. [63, 64•].

\section{Ragweed}

Short ragweed is native to Northern and Central America, where it is one of the major elicitors of type I pollen allergy. Sensitization prevalence can be up to $15.3 \%$ in the general population and the weed demonstrates high cross-reactivity with other Ambrosia spp. [65-67]. Since its introduction as ballast grain, ragweed also became a relevant allergen source in parts of Europe, Asia, and Australia [5, 68]. Global warming already substantially prolonged the ragweed pollen season and further spreading of the weed to the North is predicted by in silico models [69, 70].

The pectate lyase Amb a 1 is highly abundant in ragweed pollen and represents the dominant allergen. Based on a sensitization prevalence of $>95 \%$, the natural allergen is currently considered the marker allergen for genuine ragweed sensitization $[63,71]$. In contrast to lacking cross-reactivity to homologs from the Cupressaceae family, antibody cross-reactivity with Art v 6 from mugwort pollen is observed. However, inhibition assays and $\mathrm{T}$ cell studies primarily point at a stronger allergenic potential and sensitizing role of Amb a 1 [72, 73]. In a recent study, primary sensitizing capacity of Art v 6 was also demonstrated and the authors concluded that true cosensitization of ragweed and mugwort is generally rare [10].

Recently, a cysteine protease with structural homology to group I allergens from house dust mites was identified in ragweed and designated Amb a 11 [74••]. It represents also a major allergen since $66 \%$ of patients reacted to the molecule, which was previously "hidden" in the Amb a 1 fraction. Though the majority of allergenic reactivity is clearly attributed to Amb a 1 and isolated Amb a 11 reactivity seems rare, it could be relevant for increasing the diagnostic panel [74••]. The minor ragweed allergen Amb a 4 consists of a defensinlike domain fused with a proline-rich region with homology and IgE cross-reactivity to Art v 1 from mugwort pollen [75]. Ragweed allergens belonging to the profilin, polcalcin, and nsLTP families (Table 2) are accounting for moderate to low IgE reactivity [63]. Recent transcriptome and immunoproteome data suggest 68 and $41 \%$ IgE reactivity for ragweed polygalacturonase and enolase, respectively [71], which together with Amb a 3 might be worth investigating at the molecule level.

\section{Mugwort}

Common mugwort is endemic in the Northern Hemisphere and Australia and represents the best studied plant within the genus. Among pollinosis patients, sensitization to mugwort pollen ranges between 10 and $14 \%$ in the European and Asian population $[63,76]$. Habitat and pollination of mugwort are vastly overlapping with ragweed and thus confounding allergy anamnesis. Art v 1, consisting of a defensin-like domain fused with a proline-rich region, represents the major allergen 
with a sensitization prevalence of $70-95 \%[77,78]$. The marker allergen demonstrates partial IgE cross-reactivity with Amb a 4 from ragweed and Par h 1 from feverfew pollen. Primary sensitization seems to be predominately evolving by the major mugwort allergen (Pablos et al., unpublished data). In addition, Art $\mathrm{v} 3$ is of particular interest in regions where nsLTP-related food and pollen allergies are observed [79]. While Art v 3 might pave the way to food-related symptoms in peach allergic patients [80], recent data also suggest that it is able to bona fide elicit respiratory symptoms in patients [81, 82•]. In mugwort sensitized patients, Art v 6 in hand with Art v 1 is considered highly indicative for primary mugwort sensitization [10].

\section{Pellitory}

Pollen of pellitory is responsible for allergic reactions predominately in Mediterranean regions with sensitization frequencies reaching 60-90\% [63]. Due to climatic changes, flowering periods throughout the year were registered [83]. The main allergen components are Par $\mathrm{j} 1$ and Par j 2, both belonging to the nsLTP family showing a sensitization frequency of up to $95 \%$ [63, 84]. They are considered marker allergens for genuine pellitory allergy, since they do not crossreact with homologs from other pollen and food [85]. Par $\mathrm{j} 2$ showed high sensitivity and specificity and is currently used for routine molecule-based diagnosis of pellitory [86].

\section{English Plantain}

Plantago spp. are worldwide abundant weeds showing recurrent flowering seasons overlapping with grasses. Studies demonstrated a high clinical relevance in parts of southern and central Europe [87•, 88]. The major allergen Pla 11 belongs to the Ole e 1-like family and is recognized by $>90 \%$ of patients $[63,87 \bullet]$. Since IgE cross-reactivity with other family members is limited, it represents a highly specific marker for molecule-based diagnosis [62] (Stemeseder et al., unpublished).

\section{Russian Thistle and Goosefoot}

These weeds are considered invasive species found in arid regions of the Northern Hemisphere and Australia [62, 64•]. Due to their previous use in greening programs, they are highly abundant in Middle Eastern countries where they can represent major sensitizers for rhinitis and asthma [64•, 89, 90]. Amaranthaceae pollen also gained allergological relevance in areas of Spain, and they are expected to play an increasingly important role [91, 92]. The pectin methylesterase Sal k 1 accounts for the majority of IgE reactivity to Russian thistle and was shown to be a marker for genuine sensitization as it allows discrimination from chenopod sensitization [93, 94]. Natural glycosylated Sal k 1 is currently available for molecule-based diagnosis; however, the use of a recombinant molecule might be superior as CCDs can be avoided [95]. Sal $\mathrm{k} 5$, a member of the Ole e 1-like protein family showed $30 \%$ sensitization prevalence and considerable high IgE crossreactivity with Che a 1 from chenopod [96]. Notably, this $\mathrm{IgE}$ reactivity seems specific for Amaranthaceae and not related to Ole e 1 from olive pollen [96].

Che a 1 belongs to the Ole e 1-like family and presents a sensitization frequency of $>70 \%$ among chenopod allergic patients $[89,97]$. High IgE reactivity to chenopod profilin and polcalcin is noted, and diagnostic specificity was increased by using a cocktail of three purified proteins [89]. However, the high IgE cross-reactivity of the panallergens rather suggests that Che a 1 should be primarily considered for diagnosis although discrimination from Russian thistle sensitization seems challenging [64•, 98].

\section{Annual Mercury}

Annual mercury was reported to be an important source of pollinosis in distinct regions of Mediterranean countries [99]. For diagnostic purpose, the profilin Mer a 1 is currently available in multiplex assays; however, due to high IgE crossreactivity with other pollen profilins, interpretation of these results might be limited.

\section{Pollen Panallergens}

The term "panallergen" refers to the ubiquitous distribution of these allergens in diverse sources and profilins and polcalcins are prototypic examples [100]. The clinical relevance is considered limited, but the broad $\operatorname{IgE}$ reactivity highly influences extract-based diagnosis $[92,101 \bullet \cdot$. A panel of profilins and polcalcins, considered representatives of the entire panallergen group, is available for molecule-based diagnosis allowing discrimination between genuine and panallergen sensitization (Tables 1 and 2). For in vivo diagnosis, natural panallergens from palm tree were previously used [102]. In contrast, members of the pectate lyase, nsLTP, and Ole e 1-like families demonstrate heterogeneous reactivity pattern. IgE cross-reactivity is mostly confined to closely related molecules with high sequence identity and might vary in different populations (Fig. 1).

\section{Conclusions}

Diagnosis of pollen allergies mainly relies on careful anamnesis, which includes narrowing down the sensitizing plant by the time clinical symptoms are observed. However, flowering periods are frequently overlapping and can vary in distinct geographic regions while climatic changes are expected to further aggravate the problem $[1,69,70]$. Pollen allergic 
patients are typically reacting to more than one source, thus specific diagnosis is applied either using allergen extracts or purified components. Based on molecule-based approaches, more specific results are obtained and clinically irrelevant sensitizations due to, e.g., panallergens or other IgE crossreactive compounds can be circumvented [11]. The currently available panel of allergen components is covering most of the common allergen sources; however, in comparison to extractbased products, there is a substantial gap in quantity. Allergy research will certainly further increase the panel of relevant allergens of known sources as well as novel allergens from plants that were less explored for diagnosis [37••]. Moleculebased diagnosis can improve sensitivity, specificity, predict severity of reactions, and identify the genuine sensitizing source [103]. This refined diagnosis was recently shown to change a large proportion of allergen immunotherapy prescription as opposed to relying on anamnesis and SPT alone $[101 \bullet \cdot$. In future, allergy diagnosis using reliable biomarkers might be crucial when considering allergen immunotherapy in the context of precision medicine [104].

Acknowledgments Open access funding provided by Austrian Science Fund (FWF). The financial support by the Austrian Science Fund (I 1152, P27589, and L688), the Austrian Federal Ministry of Science, Research and Economy, the National Foundation of Research, Technology and Development, Land Salzburg, and the priority program "Allergy-CancerBioNano Research Centre" of the University of Salzburg is acknowledged.

\section{Compliance with Ethical Standards}

Conflict of Interest Dr. Gadermaier reports personal fees from Thermo Fisher Scientific. Drs. Pablos, Wildner, Asam, and Wallner declare no conflicts of interest.

Human and Animal Rights and Informed Consent This article does not contain any studies with human or animal subjects performed by any of the authors.

Open Access This article is distributed under the terms of the Creative Commons Attribution 4.0 International License (http:// creativecommons.org/licenses/by/4.0/), which permits unrestricted use, distribution, and reproduction in any medium, provided you give appropriate credit to the original author(s) and the source, provide a link to the Creative Commons license, and indicate if changes were made.

\section{References}

Papers of particular interest, published recently, have been highlighted as:

- Of importance

•- Of major importance

1. D'Amato $G$ et al. Effects on asthma and respiratory allergy of climate change and air pollution. Multidiscip Respir Med. 2015;10:39.
2. Fukuoka A et al. Diesel exhaust particles exacerbate allergic rhinitis in mice by disrupting the nasal epithelial barrier. Clin Exp Allergy. 2015.

3. Wimmer M et al. Pollen-derived adenosine is a necessary cofactor for ragweed allergy. Allergy. 2015;70(8):944-54.

4. Prado $\mathrm{N}$ et al. Pollensomes as natural vehicles for pollen allergens. J Immunol. 2015;195(2):445-9.

5. Scevkova $J$ et al. Influence of airborne pollen counts and length of pollen season of selected allergenic plants on the concentration of sIgE antibodies on the population of Bratislava, Slovakia. Ann Agric Environ Med. 2015;22(3):451-5.

6. Newson RB et al. Geographical variation in the prevalence of sensitization to common aeroallergens in adults: the GA(2) LEN survey. Allergy. 2014;69(5):643-51.

7. Panzner $P$ et al. A comprehensive analysis of middle-European molecular sensitization profiles to pollen allergens. Int Arch Allergy Immunol. 2014;164(1):74-82.

8. D'Amato G, Liccardi G. Pollen-related allergy in the European Mediterranean area. Clin Exp Allergy. 1994;24(3):210-9.

9. Hauser $\mathrm{M}$ et al. Bet v 1-like pollen allergens of multiple Fagales species can sensitize atopic individuals. Clin Exp Allergy. 2011;41(12):1804-14.

10. Asero R et al. Concomitant sensitization to ragweed and mugwort pollen: who is who in clinical allergy? Ann Allergy Asthma Immunol. 2014;113(3):307-13.

11. Ferreira F, Wolf M, Wallner M. Molecular approach to allergy diagnosis and therapy. Yonsei Med J. 2014;55(4):839-52.

12. Himly $\mathrm{M}$ et al. Standardization of allergen products: 2. Detailed characterization of GMP-produced recombinant Phl p 5.0109 as European pharmacopoeia reference standard. Allergy. 2015. Extensive physico-chemcial and immunological characterization of Phl p 5 which is now available as European Pharmacopoeia allergen reference standard for grass pollen products.

13. Niederberger V, Eckl-Dorna J, Pauli G. Recombinant allergenbased provocation testing. Methods. 2014;66(1):96-105. This paper reviews several important provocation methods and describes the earlier usage of allergen molecules in clinical provocation studies.

14. Lupinek $\mathrm{C}$ et al. Advances in allergen-microarray technology for diagnosis and monitoring of allergy: the MeDALL allergen-chip. Methods. 2014;66(1):106-19.

15. Khan FM et al. Basophil activation test compared to skin prick test and fluorescence enzyme immunoassay for aeroallergen-specific Immunoglobulin-E. Allergy Asthma Clin Immunol. 2012;8(1):1.

16. Wong V et al. Early pollen sensitization in children is dependent upon regional aeroallergen exposure. J Allergy. 2012;2012: 583765.

17. Kleine-Tebbe J. Big-time sensitization rates in young Germans: big numbers-big risks-big confusion? Int Arch Allergy Immunol. 2014;163(1):3-4.

18. Price $\mathrm{D}$ et al. The hidden burden of adult allergic rhinitis: UK healthcare resource utilisation survey. Clin Transl Allergy. 2015;5:39

19. Vorontsova MS, Clayton D, Simon BK. Grassroots e-floras in the Poaceae: growing GrassBase and GrassWorld. PhytoKeys. 2015;48:73-84.

20. Davies JM. Grass pollen allergens globally: the contribution of subtropical grasses to burden of allergic respiratory diseases. Clin Exp Allergy. 2014;44(6):790-801.

21.• Popescu FD. Molecular biomarkers for grass pollen immunotherapy. World J Methodol. 2014;4(1):26-45. This paper reviews the most important molecular biomarkers in grass pollen allergy to support accurate prescription for allergen immunotherapy. 
22. Darsow U et al. Allergens. Heterogeneity of molecular sensitization profiles in grass pollen allergy - implications for immunotherapy? Clin Exp Allergy. 2014;44(5):778-86.

23. Scaparrotta $A$ et al. Sensitization to timothy grass pollen allergenic molecules in children. Multidiscip Respir Med. 2013;8(1):17.

24.• Hatzler L et al. Molecular spreading and predictive value of preclinical IgE response to Phleum pratense in children with hay fever. J Allergy Clin Immunol. 2012;130(4):894-901. e5. Within this publication, the phenomenon "molecular spreading" which describes the sequential development of antibody response to non-cross-reacting molecules from the same source, starting with an initiator molecule was shown for grass pollen allergy.

25. Campbell BC et al. Total transcriptome, proteome, and allergome of Johnson grass pollen, which is important for allergic rhinitis in subtropical regions. J Allergy Clin Immunol. 2015;135(1):13342. This work demonstrated for the first time the presence of group II grass allergens in subtropical sources which until now were thought to be restricted to grasses of the temperate regions.

26. Andersson K, Lidholm J. Characteristics and immunobiology of grass pollen allergens. Int Arch Allergy Immunol. 2003;130(2): 87-107.

27. Devanaboyina SC et al. High-resolution crystal structure and $\operatorname{IgE}$ recognition of the major grass pollen allergen $\mathrm{Phl} \mathrm{p} \mathrm{3.} \mathrm{Allergy.}$ 2014;69(12):1617-28

28. Nandy A et al. Primary structure, recombinant expression, and molecular characterization of $\mathrm{Phl} \mathrm{p} \mathrm{4,} \mathrm{a} \mathrm{major} \mathrm{allergen} \mathrm{of} \mathrm{timothy}$ grass (Phleum pratense). Biochem Biophys Res Commun. 2005;337(2):563-70.

29. Sastre J. Molecular diagnosis in allergy. Clin Exp Allergy. 2010;40(10):1442-60.

30. Asero R et al. Prevalence and clinical relevance of IgE sensitization to profilin in childhood: a multicenter study. Int Arch Allergy Immunol. 2015;168(1):25-31.

31. Johansen $\mathrm{N}$ et al. Extensive $\mathrm{IgE}$ cross-reactivity towards the Pooideae grasses substantiated for a large number of grass-pollen-sensitized subjects. Int Arch Allergy Immunol. 2009;150(4):325-34.

32. Timbrell VL et al. An immunodiagnostic assay for quantitation of specific IgE to the major pollen allergen component, Pas n 1, of the subtropical Bahia grass. Int Arch Allergy Immunol. 2014;165(4): 219-28. This work showed improved sensitivity and specificity when using nPas $\mathbf{n} 1$ for molecular diagnosis of Bahia grass pollen allergy and the authors suggested inclusion for routine molecular diagnosis as first allergen of the Panicoideae subfamily.

33. Davies JM et al. Functional immunoglobulin E cross-reactivity between Pas $\mathrm{n} 1$ of Bahia grass pollen and other group 1 grass pollen allergens. Clin Exp Allergy. 2011;41(2):281-91.

34. Etto $\mathrm{T}$ et al. Unique and cross-reactive $\mathrm{T}$ cell epitope peptides of the major Bahia grass pollen allergen, Pas n 1. Int Arch Allergy Immunol. 2012;159(4):355-66.

35. Marth $\mathrm{K}$ et al. Human monoclonal antibody-based quantification of group 2 grass pollen allergens. J Allergy Clin Immunol. 2004;113(3):470-4.

36. Gschwantner $\mathrm{T}$ et al. Common tree definitions for national forest inventories in Europe. Silva Fennica. 2009;43(2):303-21.

37.• Asam $\mathrm{C}$ et al. Tree pollen allergens-an update from a molecular perspective. Allergy. 2015;70(10):1201-11. This comprehensive review provides an excellent description at the molecular level on all officially acknowledged tree pollen allergens.

38.• Burbach GJ et al. GA(2)LEN skin test study II: clinical relevance of inhalant allergen sensitizations in Europe. Allergy. 2009;64(10): $1507-15$. The study provides a comprehensive Europe-wide overview on allergic sensitization towards 18 inhalent allergens using skin prick tests.

39. Campana $\mathrm{R}$ et al. Hypoallergenic derivatives of the major birch pollen allergen Bet $\mathrm{v} 1$ obtained by rational sequence reassembly. $\mathrm{J}$ Allergy Clin Immunol. 2010;126(5):1024-31. 1031 e1-8.

40. Moverare R et al. Different IgE reactivity profiles in birch pollensensitive patients from six European populations revealed by recombinant allergens: an imprint of local sensitization. Int Arch Allergy Immunol. 2002;128(4):325-35.

41. Sekerkova A, Polackova M. Detection of Bet v1, Bet v2 and Bet $\mathrm{v} 4$ specific IgE antibodies in the sera of children and adult patients allergic to birch pollen: evaluation of different IgE reactivity profiles depending on age and local sensitization. Int Arch Allergy Immunol. 2011;154(4):278-85.

42. Geroldinger-Simic $\mathrm{M}$ et al. Birch pollen-related food allergy: clinical aspects and the role of allergen-specific IgE and IgG4 antibodies. J Allergy Clin Immunol. 2011;127(3):616-22. e1.

43. Price A et al. Oral allergy syndrome (pollen-food allergy syndrome). Dermatitis. 2015;26(2):78-88.

44. Rossi RE, Monasterolo G, Monasterolo S. Detection of specific IgE antibodies in the sera of patients allergic to birch pollen using recombinant allergens Bet $\mathrm{v} 1$, Bet $\mathrm{v}$ 2, Bet $\mathrm{v} 4$ : evaluation of different $\mathrm{IgE}$ reactivity profiles. Allergy. 2003;58(9):929-32.

45. Asturias JA et al. Purification and characterization of Pla a 1, a major allergen from Platanus acerifolia pollen. Allergy. 2002;57(3):221-7.

46. Chen $\mathrm{Z}$ et al. Characterization of two pollen allergens of the London plane tree in Shanghai. Iran J Allergy Asthma Immunol. 2015;14(2):139-48.

47. Ibarrola I et al. Identification of a polygalacturonase as a major allergen (Pla a 2) from Platanus acerifolia pollen. J Allergy Clin Immunol. 2004;113(6):1185-91.

48. Pazouki $\mathrm{N}$ et al. Oriental plane pollen allergy: identification of allergens and cross-reactivity between relevant species. Allergy Asthma Proc. 2008;29(6):622-8.

49. Lauer I et al. Identification of a plane pollen lipid transfer protein (Pla a 3) and its immunological relation to the peach lipid-transfer protein, Pru p 3. Clin Exp Allergy. 2007;37(2):261-9.

50. Villalba M, Rodriguez R, Batanero E. The spectrum of olive pollen allergens. From structures to diagnosis and treatment. Methods. 2014;66(1):44-54. The paper reviews the current literature of olive pollen allergens including detailed description of sensitization prevalence towards individual allergenic molecules.

51. Esteve $\mathrm{C}$ et al. Analysis of olive allergens. Talanta. 2012;92:1-14.

52. Barderas $\mathrm{R}$ et al. Cloning, expression, and clinical significance of the major allergen from ash pollen, Fra e 1. J Allergy Clin Immunol. 2005;115(2):351-7.

53. Imhof $\mathrm{K}$ et al. Ash pollen allergy: reliable detection of sensitization on the basis of IgE to Ole e 1. Allergo J Int. 2014;23(3):78-83.

54. Torres $\mathrm{M}$ et al. An enzymatically active beta-1,3-glucanase from ash pollen with allergenic properties: a particular member in the oleaceae family. PLoS One. 2015;10(7), e0133066.

55. Gonzalez E, Villalba M, Rodriguez R. Immunological and molecular characterization of the major allergens from lilac and privet pollens overproduced in Pichia pastoris. Clin Exp Allergy. 2001;31(2):313-21.

56. Scala $\mathrm{E}$ et al. Cross-sectional survey on immunoglobulin $\mathrm{E}$ reactivity in 23,077 subjects using an allergenic moleculebased microarray detection system. Clin Exp Allergy. 2010;40(6):911-21.

57. Okamoto Y et al. Present situation of cedar pollinosis in Japan and its immune responses. Allergol Int. 2009;58(2):155-62. 
58. Yoshida $\mathrm{K}$ et al. Cedar and cypress pollen counts are associated with the prevalence of allergic diseases in Japanese schoolchildren. Allergy. 2013;68(6):757-63.

59. Pettyjohn M, Levetin E. A comparative biochemical study of conifer pollen allergens. Aerobiologia. 1997;13:259-67.

60. Mori Tet al. Purification, identification, and cDNA cloning of Cha o 2, the second major allergen of Japanese cypress pollen. Biochem Biophys Res Commun. 1999;263(1):166-71.

61. Yokoyama $\mathrm{M}$ et al. Purification, identification, and cDNA cloning of Jun a 2, the second major allergen of mountain cedar pollen. Biochem Biophys Res Commun. 2000;275(1):195-202.

62. Stemeseder T et al. Marker allergens of weed pollen - basic considerations and diagnostic benefits in the clinical routine: part 16 of the series molecular allergology. Allergo J Int. 2014;23(8):274 80.

63. Gadermaier G, Hauser M, Ferreira F. Allergens of weed pollen: an overview on recombinant and natural molecules. Methods. 2014;66(1):55-66

64. Villalba $\mathrm{M}$ et al. Amaranthaceae pollens: review of an emerging allergy in the mediterranean area. J Investig Allergol Clin Immunol. 2014;24(6):371-81. quiz 2 p preceding 382. An extensive overview on Amaranthaceae pollen allergens and their physicochemical and immunological properties is provided.

65. Chan-Yeung $\mathrm{M}$ et al. Geographical variations in the prevalence of atopic sensitization in six study sites across Canada. Allergy. 2010;65(11):1404-13.

66. Bousquet PJ et al. Geographical variation in the prevalence of positive skin tests to environmental aeroallergens in the European Community Respiratory Health Survey I. Allergy. 2007;62(3):301-9.

67. Christensen LH et al. Short ragweeds is highly cross-reactive with other ragweeds. Ann Allergy Asthma Immunol. 2015;115(6): 490-5. e1.

68. Rueff $F$ et al. Sensitization to common ragweed in southern Bavaria: clinical and geographical risk factors in atopic patients. Int Arch Allergy Immunol. 2012;159(1):65-74.

69. Ziska $\mathrm{L}$ et al. Recent warming by latitude associated with increased length of ragweed pollen season in central North America. Proc Natl Acad Sci U S A. 2011;108(10):4248-51.

70. Storkey $\mathrm{J}$ et al. A process-based approach to predicting the effect of climate change on the distribution of an invasive allergenic plant in Europe. PLoS One. 2014;9(2), e88156.

71. Bordas-Le Floch V et al. Identification of novel short ragweed pollen allergens using combined transcriptomic and immunoproteomic approaches. PLoS One. 2015;10(8), e0136258.

72. Pichler $\mathrm{U}$ et al. Pectate lyase pollen allergens: sensitization profiles and cross-reactivity pattern. PLoS One. 2015;10(5), e0120038.

73. Jahn-Schmid B et al. Humoral and cellular cross-reactivity between Amb a 1, the major ragweed pollen allergen, and its mugwort homolog Art v 6. J Immunol. 2012;188(3):1559-67.

74.•• Bouley J et al. Identification of the cysteine protease Amb a 11 as a novel major allergen from short ragweed. J Allergy Clin Immunol. 2015;136(4):1055-64. Within this work, the novel IgE reactive cysteine protease Amb a 11 was identified in ragweed pollen which shares similar physico-chemical properties with the dominant allergen Amb a 1.

75. Leonard $\mathrm{R}$ et al. A new allergen from ragweed (Ambrosia artemisiifolia) with homology to art v 1 from mugwort. J Biol Chem. 2010;285(35):27192-200.

76. Li J et al. A multicentre study assessing the prevalence of sensitizations in patients with asthma and/or rhinitis in China. Allergy. 2009.

77. Himly M et al. Art v 1, the major allergen of mugwort pollen, is a modular glycoprotein with a defensin-like and a hydroxyprolinerich domain. Am Fasten J. 2003;17(1):106-8.
78. Moverare R et al. Mugwort-sensitized individuals from North Europe, South Europe and North America show different IgE reactivity patterns. Int Arch Allergy Immunol. 2011;154(2):164-72.

79. Egger $\mathrm{M}$ et al. The role of lipid transfer proteins in allergic diseases. Curr Allergy Asthma Rep. 2010;10(5):326-35.

80. Gao ZS et al. Peach allergy in China: a dominant role for mugwort pollen lipid transfer protein as a primary sensitizer. J Allergy Clin Immunol. 2013;131(1):224-6. e1-3.

81. Sanchez-Lopez $\mathrm{J}$ et al. Role of Art $\mathrm{v} 3$ in pollinosis of patients allergic to Pru p 3. J Allergy Clin Immunol. 2014;133(4):1018-25.

82. Scala E et al. Lipid transfer protein sensitization: reactivity profiles and clinical risk assessment in an Italian cohort. Allergy. 2015;70(8):933-43. Testing a large cohort of nsLTP patients revealed important association profiles and clusters and allowed drawing conclusions regarding clinical pictures.

83. Ariano R, Canonica GW, Passalacqua G. Possible role of climate changes in variations in pollen seasons and allergic sensitizations during 27 years. Ann Allergy Asthma Immunol. 2010;104(3):215-22.

84. Stumvoll $\mathrm{S}$ et al. Identification of cross-reactive and genuine Parietaria judaica pollen allergens. J Allergy Clin Immunol. 2003;111(5):974-9.

85. Tordesillas L et al. Plant lipid transfer protein allergens: no crossreactivity between those from foods and olive and Parietaria pollen. Int Arch Allergy Immunol. 2011;156(3):291-6.

86. Gonzalez-Rioja R et al. Diagnosis of Parietaria judaica pollen allergy using natural and recombinant Par $\mathrm{j} 1$ and Par $\mathrm{j} 2$ allergens. Clin Exp Allergy. 2007;37(2):243-50.

87. Gadermaier G et al. Plantago lanceolata: an important trigger of summer pollinosis with limited IgE cross-reactivity. J Allergy Clin Immunol. 2014;134(2):472-5. e5. This is the first report about the high clinical relevance of English plantain pollen allergy in a cohort of the temperate climate.

88. Sousa R et al. Identification of Plantago lanceolata pollen allergens using an immunoproteomic approach. J Investig Allergol Clin Immunol. 2014;24(3):177-83.

89. Nouri HR et al. Diagnosis of Chenopodium album allergy with a cocktail of recombinant allergens as a tool for component-resolved diagnosis. Mol Biol Rep. 2012;39(3):3169-78.

90. Farrokhi $\mathrm{S}$ et al. Common aeroallergens in patients with asthma and allergic rhinitis living in southwestern part of Iran: based on skin prick test reactivity. Iran J Allergy Asthma Immunol. 2015;14(2):133-8.

91. Barber D et al. Understanding patient sensitization profiles in complex pollen areas: a molecular epidemiological study. Allergy. 2008;63(11):1550-8.

92. Barber $\mathrm{D}$ et al. Component-resolved diagnosis of pollen allergy based on skin testing with profilin, polcalcin and lipid transfer protein pan-allergens. Clin Exp Allergy. 2009;39(11):1764-73.

93. Barderas $\mathrm{R}$ et al. A pectin methylesterase as an allergenic marker for the sensitization to Russian thistle (Salsola kali) pollen. Clin Exp Allergy. 2007;37(7):1111-9.

94. Cuesta-Herranz J et al. Differences among pollen-allergic patients with and without plant food allergy. Int Arch Allergy Immunol. 2010;153(2):182-92.

95. Mas $\mathrm{S}$ et al. A recombinant $\mathrm{Sal} \mathrm{k} 1$ isoform as an alternative to the polymorphic allergen from Salsola kali pollen for allergy diagnosis. Int Arch Allergy Immunol. 2015;167(2):83-93.

96. Castro L et al. Sal k 5, a member of the widespread Ole e 1-like protein family, is a new allergen of Russian thistle (Salsola kali) pollen. Int Arch Allergy Immunol. 2014;163(2):142-53.

97. Barderas $\mathrm{R}$ et al. Identification and characterization of Che a 1 allergen from Chenopodium album pollen. Int Arch Allergy Immunol. 2002;127(1):47-54. 
98. Sirvent $\mathrm{S}$ et al. Pollen and plant food profilin allergens show equivalent IgE reactivity. Ann Allergy Asthma Immunol. 2011;106(5):429-35.

99. Vallverdu A et al. Characterization of recombinant Mercurialis annua major allergen Mer a 1 (profilin). J Allergy Clin Immunol. 1998;101(3):363-70.

100. Hauser $\mathrm{M}$ et al. Panallergens and their impact on the allergic patient. Allergy Asthma Clin Immunol. 2010;6(1):1.

101.• Stringari $\mathrm{G}$ et al. The effect of component-resolved diagnosis on specific immunotherapy prescription in children with hay fever. $\mathrm{J}$ Allergy Clin Immunol. 2014;134(1):75-81. This excellent work shows how molecule-based allergy diagnosis is directly impacting clinicians' decision-making for immunotherapy.

102. Asero R. Hypersensitivity to pollen panallergens (profilin and polcalcin) detected in vitro and in vivo: a comparative analysis. J Investig Allergol Clin Immunol. 2011;21(4): 323-4.

103. Hamilton RG, Kleine-Tebbe J. Molecular allergy diagnostics: analytical features that support clinical decisions. Curr Allergy Asthma Rep. 2015;15(9):57.

104. Canonica GW et al. Allergen immunotherapy (AIT): a prototype of precision medicine. World Allergy Organ J. 2015;8(1):31. 